\title{
Ungünstiges Kosten-Nutzen-Verhältnis für Statine als Primärprävention bei kleinem Risiko von Herz-Kreislauf-Krankheiten
}

\author{
Das Swiss Medical Board hat neue Empfehlungen zur Anwendung von Statinen als \\ Primärprävention von Herz-Kreislauf-Krankheiten veröffentlicht. In seinem Bericht \\ kommt das Fachgremium zum Schluss, dass vor einer Verschreibung von Statinen \\ das individuelle Risiko für ein kardiovaskuläres Ereignis abzuklären ist und andere \\ Möglichkeiten zur Risikoreduktion auszuschöpfen sind. Falls das Risiko für kardio- \\ vaskuläre Erkrankung weniger als $10 \%$ beträgt, ist der Einsatz von Statinen nicht \\ angezeigt. Die Trägerschaft hat den Bericht zur Kenntnis genommen und unter- \\ breitet inn einer Post-Publication Review.
}

Interaktiver
Artikel
Wollen Sie diesen Artikel
kommentieren? Nutzen
Sie daür die Kommentar-
funktion in der Online-
Version oder sehen Sie
nach, was Ihre Kolleginnen
und Kollegen bereits
geschrieben haben:
www.saez.ch/
aktuelle-ausgabe/
interaktive-beitraege/

Korrespondenz:

Susanna Marti Calmell Sekretariat Trägerschaft Swiss Medical Board Stampfenbachstrasse 30 CH-8090 Zürich Tel. 0432595211

info[at]medical-board www.swissmedicalboard.ch
Unter dem Begriff «Statine» werden Arzneimittel zusammengefasst, die bei der Behandlung zu hoher Cholesterinspiegel im Blut zum Einsatz kommen. Cholesterin ist ein wichtiger Bestandteil von Zellmembranen; entsprechend ist das Vorhandensein von Cholesterin eine Voraussetzung für eine normale Körperfunktion. $\mathrm{Zu}$ hohe Cholesterinspiegel stellen jedoch neben anderen Faktoren wie z. B. Rauchen und Bluthochdruck einen Risikofaktor für kardiovaskuläre Erkrankungen dar. Die Behandlung mit Statinen bei Patientinnen und Patienten, die bereits an einer kardiovaskulären Erkrankung leiden (z.B. koronare Herzerkrankung) oder gar einen Herzinfarkt erlitten haben, ist international weitgehend unbestritten.

In seinem neuen Bericht geht das Fachgremium des Swiss Medical Board der Frage nach, ob eine Verschreibung von Statinen bei Personen, die nicht an einer diagnostizierten kardiovaskulären Erkrankung leiden (sogenannte «Primärprävention»), einen Einfluss hat auf die Anzahl möglicher künftiger kardiovaskulärer Ereignisse, auf die Überlebensdauer und auf die Lebensqualität.

Die untersuchte Literatur lässt den Schluss zu, dass Statine (d.h. die Senkung des Cholesterinspiegels) das Risiko für ein zukünftiges kardiovaskuläres Ereignis auch bei Personen zu reduzieren vermögen, bei denen kein kardiovaskuläres Leiden diagnostiziert ist. Allerdings ist ein hoher Cholesterinwert nur einer von vielen Risikofaktoren; weitere sind z.B. Rauchen, Übergewicht oder Bluthochdruck. Bei diesen gilt es als Erstes anzusetzen, da die Einnahme von Statinen auch mit unerwünschten Wirkungen verbunden sein kann; am häufigsten sind dies Veränderungen der Leberwerte und Muskelschädigun- gen. Zusammen mit dem hohen Preis der Statine führt dies bei Personen mit einem niedrigen Risiko für ein künftiges kardiovaskuläres Ereignis zu einem ungünstigen Kosten-Wirksamkeits-Verhältnis für die Statinbehandlung in der Primärprävention.

Das Fachgremium kommt somit zu folgenden Empfehlungen:

- Eine Verschreibung von Statinen zur Primärprävention soll erst in Betracht gezogen werden, wenn die anderen Möglichkeiten zur Reduktion der Risikofaktoren ausgeschöpft sind.

- Das Risiko für ein kardiovaskuläres Ereignis muss individuell gemäss den gültigen Guidelines bestimmt werden.

- Eine Verschreibung von Statinen in der Primärprävention ist bei einem Risiko für ein tödliches kardiovaskuläres Ereignis von unter 10\% (gemäss ESC-Score) nicht indiziert.

Post-Publication Review zum Fachbericht "Statine zur Primärprävention kardiovaskulärer Erkrankungen»

Der Fachbericht gibt die auf aktueller medizinischer Evidenz basierende Einschätzung des unabhängigen Fachgremiums Swiss Medical Board wieder. Die Trägerschaft des Swiss Medical Board hat den Bericht zur Kenntnis genommen und möchte interessierte Kreise zu einer Fachdiskussion im Sinne einer Post-Publication Review einladen. Stellungnahmen und Diskussionen können auf der Internetseite des Swiss Medical Board aufgeschaltet werden. Ihren Beitrag nehmen wir gerne entgegen unter: info[at] medical-board.ch 